\title{
Mutazioni di BRAF V600E nel craniofaringioma papillare
}

\author{
Salvatore Cannavò ${ }^{1}$
}

Pubblicato online: 19 settembre 2016

(c) Springer International Publishing AG 2016

\section{Commento a:}

\section{BRAF V600E mutations in papillary} craniopharingioma.

\section{P.K. Brastianos, S. Santagata.}

\section{Eur J Endocrinol (2016) 174:R139-R144}

Priscilla Brastianos e Sandro Santagata hanno recentemente pubblicato sullo European Journal of Endocrinology una review sulle mutazioni di BRAF V600E nei craniofaringiomi papillari, variante riscontrata soprattutto nei pazienti adulti. Sebbene siano neoplasie tipicamente benigne, i craniofaringiomi causano conseguenze cliniche a volte drammatiche e tendono a recidivare e a crescere, comportando rischi concreti anche per la sopravvivenza dei pazienti. La terapia di prima linea è generalmente chirurgica, ma il reintervento e/o il trattamento radioterapico rappresentano una frequente necessità anche per l'inefficacia delle terapie mediche tentate fino ad oggi.

La classificazione istologica dei craniofaringiomi distingue la variante papillare da quella adamantinomatosa, riscontrata sia nei bambini che negli adulti. La distinzione fra queste due varianti e la diagnosi differenziale con altre lesioni espansive della regione ipotalamo-ipofisaria (per esempio le cisti della tasca di Ratke) sono facili quando si dispone di sufficiente tessuto, ma possono diventare estremamente complesse quando il prelievo è insufficiente. In questi casi un approccio diagnostico che sfrutti le caratteristiche geniche della neoplasia può essere di grande ausilio. Infatti, il 90\% dei craniofaringiomi adamantinomatosi esprime mutazioni del gene CTNNB1, mentre una percentuale sovrapponibile di quelli papillari esprime mutazioni di BRAF V600E.

$\bowtie$ S. Cannavò

cannavos@unime.it

1 Dipartimento di Medicina Clinica e Sperimentale, Università di Messina, Messina, Italia
La mutazione di BRAF causa la sintesi di una proteina che si localizza non solo a livello citoplasmatico ma anche nucleare. Lo sviluppo di anticorpi diretti contro la proteina BRAF V600E mutata, utilizzabili in immunoistochimica, rappresenta un importante aiuto per il patologo, non solo nella distinzione fra le due varianti di craniofaringioma ma anche per differenziare il craniofaringioma papillare dalla cisti della tasca di Ratke, che ovviamente non esprime mutazioni di BRAF. Per aumentare la specificità diagnostica, a causa di possibili falsi positivi determinati dalla cross-reazione degli anticorpi con elementi di cellule sane (per esempio dell'ipofisi normale), gli autori propongono di utilizzare la genotipizzazione di BRAF, direttamente o in associazione con l'approccio immunoistochimico.

Il riscontro di mutazioni di BRAF apre la strada anche a nuove possibilità terapeutiche, analogamente a quanto avvenuto in altri tumori con le stesse mutazioni, come lo xantoastrocitoma pleiomorfo o l'ameloblastoma. A tal proposito, gli autori riportano la loro più che positiva esperienza in un caso drammatico trattato con dabrafenib, prima da solo e poi in associazione con trametinib (un inibitore di MEK che si è dimostrato capace di contrastare l'eventuale resistenza al dabrafenib e che potrebbe migliorare la risposta terapeutica a lungo termine).

Non meno interessante è che la mutazione di BRAF V600E sarebbe stata riscontrata anche nei leucociti del paziente trattato dagli autori. Se tale evidenza trovasse conferma in un'ampia casistica, si aprirebbe infatti la strada verso la possibilità di una diagnosi prechirurgica del craniofaringioma papillare.

In conclusione, la combinazione di procedure diagnostiche con miglior sensibilità e specificità e di un'efficace target therapy potrebbe ispirare l'individuazione di un nuovo algoritmo nei pazienti con sospetto craniofaringioma, che ridurrebbe i rischi e migliorerebbe gli esiti del trattamento. 